\title{
Premisas para una estrategia de gobierno electrónico en la gerencia de la virtualización universitaria
}

\author{
Piñero Martín, Ma. Lourdes* \\ Carrillo Vásquez, Alfonso** \\ García G, Blanquita C. ${ }^{* \star}$
}

\section{Resumen}

En el presente artículo se plantean reflexiones y premisas para configurar al gobierno electrónico como una estrategia de gestión de la virtualización de la Educación Superior apoyada en el uso de las TIC, más allá de la informatización e incorporación de valor a las funciones universitarias, especialmente hacia la búsqueda del mejoramiento de la calidad en la prestación de los servicios y su vinculación con la sociedad. Se propone el desarrollo, elaboración e implantación de un proyecto institucional estratégico de innovación tecnológica para el gobierno electrónico teniendo en cuenta que la gestión social del proceso de transformación de la cultura organizativa es tanto o más importante que la gestión técnica El trabajo con enfoque metodológico teórico-documental, se desagrega del proyecto de investigación "Las tecnologías de la información y la comunicación como herramienta de innovación en la gestión universitaria", adscrito a la Línea de investigación Tecnologías de información y comunicación, docencia e innovación, en la UPEL-IPB; relacionado a su vez con proyectos del Programa "Educación y Calidad de Vida" de LUZ Punto Fijo y el proyecto "Las TIC y la modernización universitaria" de la UNEXPO-Barquisimeto.

Palabras clave: Gobierno electrónico, virtualización universitaria, tecnologías de la información y la comunicación.

Recibido: 23-04-07. Aceptado: 08-10-07

Lic. en Educación. Mención Cs. Sociales. MSc. en Planificación y Gerencia en Ciencia y Tecnología .Doctora en Ciencias Mención Investigación. Docente ordinaria de la Universidad Pedagógica Experimental Libertador-Instituto Pedagógico de Barquisimeto. E-mail: malopima@cantv.net

** Profesor de Educación Industrial. Mg.Sc. en Andragogía. Docente Asociado en el área de Dibujo Industrial de la UNEXPO. E-mail: alfonsocarrillo @ cantv.net

*** Dra. en Ciencias Mención Investigación. M.Sc. en Planificación y Gerencia de Ciencia y Tecnología. Lic. en Educación, Profesora Titular, Dedicación Exclusiva en la Universidad del Zulia, Núcleo Punto Fijo. E-mail: blangar@cantv.net. 


\title{
Premises for an Electronic Government Strategy in the Management of University Virtualization
}

\begin{abstract}
This article proposes thoughts and premises to configure e-government as a strategy for managing the virtualization of higher education, supported by the use of ICTs, beyond computerization and the incorporation of value in university functions, especially directed to the quest for quality improvement in service delivery and its connection with society. The study proposes the development, design and implementation of a draft for a strategic institutional project in technological innovation for e-government, taking into account that social management of the transformation process for organizational culture is as or more important than technical management. The study, with a theoreticaldocumentary methodological approach, relates to the research project, "Information and communication technologies as tools for innovation in university management," registered under the line of investigation Information and communication technologies, teaching and innovation, in the UPELIPB; and is related as well to projects of the Program "Education and Quality of Life" at LUZ, Punto Fijo, and the project "ICTs and university modernization" at UNEXPO-Barquisimeto.
\end{abstract}

Key words: Electronic government, university virtualization, information and communication technologies.

\section{Introducción}

El mundo actual enfrenta inevitablemente un impetuoso proceso de cambio que incide en casi todas las áreas y estructuras de la sociedad, sin importar cuál sea su nivel socio económico. Este proceso de cambio es cada vez más acelerado, debido al creciente, constante, innovador e ininterrumpido avance de las tecnologías de la información y comunicación (TIC), las cuales requieren y dan la pauta para una mejor velocidad de respuesta, de parte de la multiplicidad de instituciones que hacen vida en la estructura de la sociedad.

Las premisas anteriores refieren un ámbito general, sin embargo, el presente trabajo centrará su radio de alcance en la Educación Superior, la Gerencia Universitaria y las tecnologías de la información y comunicación, de forma especial en el gobierno electrónico. Las referidas varia- bles conforman un epicentro de acción que es clave en el desarrollo de cualquier país o región.

Cabe destacar que dentro de la dimensión mundial y para el presente trabajo, la variable educación es considerada de alto peso específico, siendo pilar fundamental del desarrollo general de un país. Entonces, alguien podría objetar y preguntar ¿dónde queda entonces la salud? La reflexión es válida ya que la salud es un bien intangible, no tiene precio y es también un pilar incuestionable en el desarrollo y en el mejoramiento de la calidad de vida. Sin embargo, ¿cómo se conserva y se mantiene la salud sin la educación? Obviamente, no habría desarrollo en salud sin desarrollo en educación, sería imposible hablar de investigación, innovación, gestión, generación y construcción del conocimiento sin ese pilar tan importante como lo es la Educación. 
Por su parte, la introducción y desarrollo de las tecnologías de la información y las comunicaciones plantean un reto de crucial relevancia al mundo educativo universitario. Reto que no sólo tiene que ver con la estratégica posición que la actual revolución tecnológica concede a la educación en general y a la universidad en particular, reforzada además por la aparición en el mercado de trabajo de nuevos fenómenos tales como inéditos contenidos ocupacionales, yacimientos vírgenes de empleo, la instauración de la formación continua, a los que la institución universitaria debe hacer frente; sino que también pasa por las propias posibilidades que las Tecnologías de la Información y Comunicación conceden a la innovación en la instrucción educativa.

Asimismo al desarrollo de las tecnologías de la información y comunicación se añade a los mencionados, la creación de nuevos espacios integradores para las acciones de educación superior, entornos de comunicación que establecen nuevas formas de interacción entre los miembros de la comunidad académica intra e interinstitucional, y con la ciudadanía en general. Cabe preguntarse entonces, qué rol deben desempeñar estos avances tecnológicos y comunicacionales dentro de todas las dimensiones del proceso educacional universitario que permitan la construcción de espacios de mayor eficiencia, calidad y excelencia de las funciones universitarias.

La armonización de las variables Educación Superior y Gerencia Universitaria en el escenario del Gobierno electrónico, se traduce en la aplicación de las tecnologías de la información y comunicación que permita mejorar la interacción entre los ciudadanos y el gobierno, para simplificar procesos y así, llevar el vocablo democracia más cerca del ciudadano común. En muchos países el éxito del gobierno electrónico se ha definido como éxito de la democracia electrónica. De acuerdo a Backus (2001), los objetivos de la democracia electrónica como meta superior del gobierno electrónico, se resumen en brindar acceso a los ciudadanos a la información y el conocimiento sobre los procesos políticos, los servicios y las opciones disponibles, con el objetivo de motivar la participación activa de los ciudadanos.

El concepto de universidad virtual que se viene implementando en los últimos años cada vez con mayor auge, ayuda a responder a los desafíos que han de enfrentar los universitarios, lo cual supone la utilización de las Tecnologías de la Información y Comunicación, sumado a una combinación en "justa proporción" de las diferentes herramientas tecnológicas con miras a un cambio radical innovativo en la hasta ahora, "manera de hacer las cosas".

Desde esta perspectiva, la innovación implica un sistema de educación superior al servicio de la imaginación y de la creatividad, lo cual representa promover no sólo la transformación del currículo y planes de estudios, de los métodos de enseñanza-aprendizaje, asimilación y transformación, de hacer investigación y de vincularse con el entorno, sino también una innovación en los procesos, procedimientos e información asociados con la administración y gestión de las funciones primarias universitarias. Tal como plantea la UNESCO (1997), todos los aspectos relacionados con la calidad, más 
una buena dirección, un buen gobierno y una buena administración, determinan el funcionamiento de la Universidad y la imagen institucional que proyecta a la sociedad en general.

Por ello, en el marco del uso cada día más exigente de las tecnologías de la información y comunicación, las Instituciones de Educación Superior deben ser dotadas de formas organizativas y de gestión pensadas para las prácticas pedagógicas en la virtualidad, ya que pretender replicar el modelo clásico presencial de gerencia y organización universitaria puede llevar, a corto plazo, al fracaso de proyectos no innovadores.

De ahí que un proyecto de gobierno electrónico puede constituirse en una interesante posibilidad como estrategia de innovación, transformación y modernización en la gerencia de la virtualización de la educación universitaria, que va más allá de lo tecnológico, por cuanto se introducen nuevos valores comunicacionales, de cooperación y participación interactiva entre los actores de la comunidad universitaria y la comunidad en general. Asimismo ofrece la oportunidad de modificar las estrategias de transparencia, desempeño y rendición de cuentas al interior de las administraciones, para que éstas actúen más eficientemente en la prestación de servicios y en la administración de la información, reforzando su liderazgo ante la sociedad.

El propósito del presente trabajo es, por tanto, llevar a cabo una aproximación a esta cuestión, desde una perspectiva metodológica teórico documental (UPEL, 2003), que contemple la iniciativa de gobierno electrónico como parte fun- damental de los procesos de modernización, transformación e innovación universitaria y planteando desde este contexto, algunas premisas estratégicas para su implementación. Para ello, se abordarán los siguientes aspectos:

- Impacto de las TIC en el entorno educativo universitario

- Mas allá de la virtualización de la enseñanza en las instituciones de Educación Superior

- El gobierno electrónico y la modernización de la gerencia pública

- La gerencia de la virtualización universitaria: premisas desde una estrategia de gobierno electrónico.

- Reflexiones de cierre

Asimismo, el trabajo del cual se desagrega el presente artículo, forma parte fundamental del Proyecto "Las tecnologías de la información y la comunicación como herramientas de innovación en la gestión universitaria" del Departamento de Formación Docente de la UPEL-IPB, adscrito a la línea de investigación $T e c-$ nologías de información y comunicación, docencia e innovación, en la UPEL-IPB, vinculado a su vez con el Proyecto "Las TIC y la modernización universitaria" de la UNEXPO-Barquisimeto y con los proyectos del Programa CONDES "Educación y Calidad de Vida en Paraguaná" del Núcleo LUZ Punto Fijo.

\section{Impacto de las TIC en el entorno educativo universitario}

En la actualidad, se vive un proceso histórico de evolución social a partir de lo que se dio por llamar en la década de 
los ochenta como las nuevas tecnologías de la información y las comunicación. Este fenómeno tecnológico trajo como consecuencia la configuración de una nueva estructura social y en términos generales, un nuevo tipo de sociedad a la que se le ha etiquetado como "sociedad informacional" o "sociedad de la información", la cual ha evolucionado hacia la denominada "sociedad del conocimiento" cuyo modo de desarrollo y estructuración social está basado en la nueva matriz tecnológica fundamentada en el uso intensivo de las TIC.

Se denominan Tecnologías de la Información y las Comunicación (en adelante: TIC) al conjunto de tecnologías que permiten la adquisición, producción, almacenamiento, tratamiento, comunicación, registro y presentación de informaciones, en forma de voz, imágenes y datos contenidos en señales de naturaleza acústica, óptica o electromagnética. Las TIC incluyen la electrónica como tecnología base que soporta el desarrollo de las telecomunicaciones, la informática y el audiovisual; entre las características que las distingue se puede mencionar que son: interactivas, instantáneas, innovadoras, digitales, automáticas, interconectadas, diversas y presentan altos parámetros de calidad de imagen y sonido.

EI paradigma de las TIC son las redes informáticas. De hecho, las computadoras personales de manera aislada, ofrecen una gran cantidad de posibilidades, pero conectadas incrementan su funcionalidad en varios órdenes de magnitud. Formando redes, estos equipos no sólo sivven para procesar información almacenada en soportes físicos (disco duro, disquetes, CD ROM, memorias, en- tre otros) en cualquier formato digital, sino también como herramienta para acceder a información, a recursos y servicios prestados por computadores remotos, como sistema de publicación y difusión de la información y como medio de comunicación entre seres humanos. Todo ello ha hecho de Internet un fenómeno con el que, actualmente es preciso contar en todas las esferas de la actividad humana.

Es el amplio uso de la red de Internet, lo que dinamiza la sociedad de la información hacia la complejización de fenómenos que van más allá de lo tecnológico como son la globalización, la virtualización, la interactividad y la construcción de nuevos espacios colectivos. Tal universalización de las relaciones sociales, de la información y del conocimiento es lo que a su vez Castells (1997) Ilama "La Sociedad Red".

Las consecuencias de este nuevo paradigma están provocando continuas transformaciones en las estructuras económicas, sociales y culturales. Su gran impacto en todos los ámbitos de la vida hace difícil que se pueda actuar eficientemente prescindiendo de ellas: el mundo laboral, la sanidad, la gestión económica o burocrática, el diseño industrial o artístico, la comunicación interpersonal, el comercio, la información, la calidad de vida o la educación, han generado nuevas formas de organización del trabajo, la aparición de mercados emergentes, de nuevos contenidos y perfiles ocupacionales, entre otros.

Siendo la información y el conocimiento ejes centrales de la nueva sociedad emergente, no cabe duda que el sustento que los alimenta y mantiene sea la 
triada que forman los vectores del aprendizaje, la instrucción y la educación. No es de extrañar entonces, que las instituciones de educación superior estén apostando decididamente por las TIC a partir de revisión y replanteamiento de los modelos educativos implementados hasta ahora. Es por ello que la introducción y desarrollo de las TIC plantean nuevos retos de crucial relevancia al mundo educativo universitario que obligan a asumir y decidir una diversidad de respuestas y alternativas; tales retos son para Rama (2005) equiparables a los "shocks" denominados por Alvin Toffler, al referir que:

El impacto de las nuevas tecnologías, está cambiando el panorama global, permitiendo acortar las distancias al expandir la educación fronteriza, generar la educación virtual y viabilizar no sólo una práctica pedagógica y una educación no presencial, sino la expansión de la sociedad del conocimiento asociada a la autopista de la información para todos los intangibles, y destacadamente, para la Educación Superior (p. 189190).

Es por ello que un número cada vez mayor de universidades en todo el mundo está exigiendo la alfabetización electrónica como uno de los requisitos en sus exámenes de acceso y de graduación, por considerar que es un objetivo esencial preparar a los futuros profesionales para la era digital en los centros de trabajo.

Destaca, tal como lo plantean García y Pinto (2005) que las universidades por mandato de la ley, tienen el compromiso y la obligación de formar el potencial humano que con visión de futuro será el encargado de dirigir el país en todos los ordenes: salud, educación y economía.
Por lo cual el profesor universitario debe contar con un perfil profesional actualizado, innovador y creativo, en consonancia con los nuevos retos a los que las TIC la obligan, que a su vez, le permita el desarrollo de su misión con criterios de calidad y excelencia, cumpliendo, dentro de éstos parámetros, sus funciones básicas de docencia, investigación, extensión y producción.

En este sentido, resulta innegable que las funciones referidas, conforman una cadena de referencia indispensable para garantizar la misión, visión y valores de la universidad que debe estar en armonía con los vertiginosos y acelerados cambios que tienen lugar en el mundo actual, donde las TIC, ofrecen la garantía de posibilidades para identificar, analizar y resolver los problemas y desafíos de preparación en la nueva era digital.

Las respuestas a estos desafíos se han venido gestando en los últimos 10 ó 15 años, y en la actualidad se puede evidenciar que la expresión y los perfiles de la universidad han cambiado considerablemente. Es así como la masificación, la redefinición de las relaciones enseñanza aprendizaje, la formación permanente y el aprendizaje a distancia aparecen como algunos de los factores dominantes de esta transformación. La universidad se encuentra ante nuevos procedimientos incorporados a los quehaceres académicos tradicionales, tales como, el aprendizaje en línea o e-learning, aprendizaje colaborativo, autoeducación, autoformación, aulas virtuales, tele clases o tele conferencias, redes de aprendizaje, redes de investigación, bibliotecas digitales, revistas académicas electrónicas, entre otros. 
Efectivamente al desarrollo de las TIC se añade a los mencionados a partir de la creación de nuevos espacios integradores para las acciones de educación superior, entornos de comunicación que establecen nuevas formas de interacción entre los miembros de la comunidad académica. Con las redes telemáticas es posible que esta interacción se produzca de forma sincrónica (mediante la videoconferencia o a través del chat) o bien asincrónica (mediante el correo electrónico o el foro de discusión).

Esta forma de comunicación entre los actores académicos deviene de la interactividad, como fenómeno distintivo incorporado a través del Internet el cual permite que junto a la realidad física, aparezca una nueva realidad, la virtual. Lo que ha dado lugar a la tendencia cada vez más creciente de la conformación de espacios virtuales, comunidades académicas virtuales o campus virtuales.

La dimensión interactiva se propaga desde el ambiente de aprendizaje al entorno institucional, dándoles un nuevo carácter a las instituciones de educación superior: universidad interactiva o universidad virtual. Tal como refiere Rumble (1998, cit por Angulo, 2000) “...El basarse en los valores académicos tradicionales que embonan bien con los valores de las organizaciones postburocráticas, proporcionan la base para el surgimiento de una nueva clase de universidad. Usando el término preferido de Hecksher, organización interactiva, misma que yo llamo universidad interactiva".

Efectivamente, según la UNESCO (1998) el concepto de universidad virtual ayuda a responder a los desafíos que han de enfrentar los universitarios, supone la utilización de las TIC y una combinación en "justa proporción" de las diferentes herramientas tecnológicas con miras a un cambio radical de la ecuación del costo de la educación. La pedagogía que acompaña al nuevo paradigma tecnológico permite una visión participativa de la formación que favorece un aprendizaje asincrónico, una nueva relación entre los actores y una formación a lo largo de toda la vida.

Asimismo, Silvio (1998) señala que las universidades de los países en desarrollo enfrentan el desafío de servir a una población cada vez mayor de estudiantes, más diversificada social y culturalmente, en un nuevo ambiente social, más dinámico y turbulento. De esta manera la virtualización (parcial o total) de estas organizaciones, señala el mencionado autor, puede ser un factor transformador de sus estructuras y funciones, un instrumento para mejorar su cobertura, calidad, pertinencia, equidad de acceso y una manera de construir una nueva identidad en la sociedad del conocimiento.

En este orden de ideas, conviene destacar que la virtualización de la universidad, se transforma en una nueva identidad que participa en la sociedad del conocimiento y que a su vez, requiere una nueva cultura, que consolide la prioridad del ser humano donde el cambio, la innovación, la nueva forma de sentir, pensar, actuar, la generación del conocimiento y el imperativo tecnológico de usarlos, vaya en consonancia con los valores éticos y morales, y con la generación de la capacidad de transformar los conocimientos dentro de un referencial de valores. 
Tal como lo expresa Lavados (2001; referido por García y Pinto, 2005) la potestad de convertir la información en conocimiento es una de las funciones tradicionales de la universidad a través de la investigación. Esta labor, sin embargo, también pueden realizarla otras instituciones. Pero, la utilización del saber, requiere de la convergencia de disciplinas científicas y humanistas y de una capacidad académica que sólo tiene la universidad.

\section{Más allá de la virtualización de la enseñanza en las instituciones de educación superior}

Lo virtual en el ámbito educativo aparece como un nuevo paradigma que puede ser aprovechado para potenciar el ser universitario, pero también como ocasión para la renovación institucional y como referencia clave del proceso de sociohumanización que ha tenido en la universidad un escenario privilegiado.

El impacto de las TIC planteado anteriormente concurre ahora para explicar la aparición del Aprendizaje Virtual y las Universidades Virtuales que representan formas de aprendizaje marcadamente diferentes a las utilizadas en los inicios de la educación a distancia.

Pero, ¿qué es virtualizar la universidad? Se asume que la virtualización de una organización es un proceso y resultado al mismo tiempo del tratamiento y de la comunicación mediante computadora, de datos, informaciones y conocimientos, lo cual consiste en representar electrónicamente y en forma numérica digital, objetos y procesos que se encuentran en el mundo real.
En el contexto de la educación superior, siguiendo a Silvio (2000), la virtualización está referida a la representación de procesos y objetos asociados a actividades de enseñanza y aprendizaje, de investigación y gestión, así como objetos cuya manipulación permite al usuario, realizar diversas operaciones a través de Internet, tales como aprender mediante la interacción con cursos electrónicos, inscribirse en un curso, consultar documentos en una biblioteca electrónica, y comunicarse con estudiantes y profesores. Todo ello, mediante el espacio en red que ha creado Internet.

Al respecto, Silvio (1998) señala que la nueva configuración de los espacios virtuales en la universidad deviene en la concepción de un campus virtual cuyo esquema podría vincular y soportar espacios funcionales virtualizados: en el aula virtual, la transferencia de conocimiento; en el laboratorio virtual, la generación de conocimiento; en la biblioteca virtual, la conservación e intercambio de conocimiento, y en la oficina virtual, la gestión general del conocimiento. Todo lo cual implica apoyar tecnológicamente los procesos universitarios hasta potenciar su virtualidad tales como la enseñanza/aprendizaje, la investigación, la interacción con el entorno, la gestión y la codificación y recuperación de información.

De allí que, virtualizar la universidad es sobre todo virtualizar sus espacios funcionales, esto es, disponer sectores del ciberespacio para apoyar o sustituir tecnológicamente las actividades académicas y administrativas realizadas físicamente en los espacios tradicionales, de modo que su virtualidad, en los términos arriba descritos, se potencie. 
En Venezuela, son muchas las universidades que ya han iniciado o están en franco proceso de avance del proceso de virtualización educativa, especialmente dirigido hacia los procesos vinculados con la docencia. Por lo que no es raro encontrar como el sistema educativo superior ofrece a través de sus portales una gran disparidad de desarrollo en plataformas de gestión del conocimiento, también denominadas plataformas educativas, que se constituyen como programas integrados (cursos en línea, diplomados, cursos de pregrado, programas de postgrado asignaturas, tutoriales) con los consecuentes componentes de contenidos sustantivos educativos y sus correspondientes materiales, mecanismos de evaluación, herramientas de comunicación (como el correo electrónico o los foros), así como instrumentos a disposición del alumno para que desarrolle sus potenciales capacidades de autoaprendizaje y maduración cognitiva e intelectual.

Asimismo, la mayoría de las universidades venezolanas poseen portales en la web, en los cuales es posible apreciar que ofrecen distintos grados de virtualización, pues existen casos de Universidades que tienen su sede física pero además ofrecen algunos cursos virtuales. $Y$ por otro lado existen instituciones de educación superior que no sólo ofrecen un curso o materiales virtuales, sino brindan también servicios virtuales como el de bi- blioteca, inscripción, consulta de expedientes, calificaciones, asesorías, centros de investigación e incluso a veces hasta chats estudiantiles, espacios donde pueden los alumnos interrelacionarse de manera informal ${ }^{1}$.

De igual modo, puede decirse de la investigación en la universidad que, gracias a las TIC, gana en agilidad y rapidez en los trámites administrativos y organizativos de gestión de los proyectos, en la posibilidad de acceder en línea a bases de datos, documentación y convocatorias, en la capacidad de hacer posible la participación real en proyectos intra e inter universitarios (antes una posibilidad más bien formal con poco grado de operatividad), amén de permitir la creación de una red mundial (habitualmente mediante el correo electrónico y foros específicos) con posibilidad de materializar en tiempo real los procesos de comunicación y validación del conocimiento científico.

Surge la inquietud de preguntar: ¿cómo hubiese resultado, otrora, las funciones de la universidad si se hubiese contado con las actuales TIC y de forma especial con la posibilidad de una gestión con la presencia del gobierno electrónico? La respuesta no se hace esperar, de seguro una revolución científica, social, educativa, política, religiosa y cultural sin precedentes, con nuevos paradigmas de los cuales hoy seríamos los herederos.

1 Para mayor información, recomendamos la revisión del documento Diagnóstico de la Educación Virtual en Venezuela de Curci La Rocca (2003).Documento en línea, disponible en: http: //www.iesalc.unesco.org.ve/programas/internac/univ_virtuales/venezuela/vir_ve.pdf\#search= $\% 22$ educacion $\% 20$ virtual $\% 20$ en $\% 20$ venezuela $\% 22$. 
Sin embargo, la realidad es que a la universidad le toca dejar en herencia caminos de excelencia apoyados por las TIC.

A este conjunto de facetas vinculadas a la docencia, investigación y extensión puede sumarse la integración de las tareas de gestión propiamente dichas, dando lugar a la creación de campus virtuales que, como un ejemplo llamativo, permite la matrícula a través de Internet, eliminando con ello situaciones tan incómodas como las interminables colas y el sinfín de aglomeraciones que en torno a las secretarias de los centros de enseñanza se producen todos los comienzos de cursos. En este aspecto, vale decir que son muy escasas las experiencias de universidades venezolanas que han virtualizado completamente dichos procesos, y por lo general lo combinan con la presencialidad.

En un estudio realizado por Curci La Roca (2003) sobre el Diagnóstico de la Educación virtual en Venezuela refiere que "no existe en el país hasta los momentos una Universidad Virtual creada únicamente para ese fin"(pág 27). Asimismo señala que para la fecha, el estudio se realizó a una población de 42 universidades, de las cuales el 16 (38\%) de ellas tienen actualmente programas académicos virtuales, algunos consolidados y otros en sus comienzos, de las restantes 26 instituciones, 16 (38\%) no tienen en los actuales momentos y 10 (24\%) tienen proyectos planteados a futuro. Igualmente, de las 16 Universidades con proyectos académicos virtuales, 7 (44\%) son privadas y 9 (56\%) son oficiales (idem).

Por otro lado, llama la atención la variedad de los resultados en cuanto a las otras estructuras, procesos y espacios u objetos que se encuentran en línea, además de los programas académicos virtuales ofrecidos. Entre los cuales resaltan los bajos porcentajes presentes en los procesos y servicios vinculados con la "gestión de la educación virtual"; a saber (pág 42-43):

- Noticias e información: $89 \%$

- Inscripciones: $74 \%$

- Pruebas de ingreso a la institución: $11 \%$

- Pagos de matrículas y de otros servicios académicos: $42 \%$

- Planeación Académica de los Programas (determinación de requisitos, créditos, asignación de docentes, salones, horarios, cargas académicas, etc.): $63 \%$

- Elaboración y registro académico de los programas de estudio por parte de los estudiantes: $32 \%$

- Histórico de notas de los estudiantes: $42 \%$

- Enlaces permanentes con bases bibliográficas: $68 \%$ Biblioteca virtual propia (se han digitalizado y puesto al servicio los textos fundamentales para consulta virtual): $47 \%$

- Secretaría Académica Virtual (para la solicitud y elaboración de certificados, trámites, elaboración de cartas y etiquetas, etc.: $11 \%$

- Gestión de Docentes (para administración de los horarios, cargas de los docentes, tipo de funciones que realiza, etc.): $37 \%$

- Planeación y gestión financiera de los programas y de la institución: $21 \%$

- Manejo de los programas como Centros de Costos: $11 \%$ 
- Elaboración de Reportes Estadísticos y tablas: $37 \%$

- Servicios de Banca virtual: $16 \%$

- Comercio electrónico: $11 \%$

En relación a este último aspecto, es necesario considerar el papel que las TIC han operado en la transformación sobre los procesos de gestión académica y administrativa, a través de las respectivas unidades de gestión siendo las primeras que de manera rutinaria incorporaron en sus tareas innovaciones generadas por estas herramientas. Tal como lo expresa (Torres Alvero, s.f.),

(...) su aplicación mediante los nuevos recursos telemáticos constituye un claro ejemplo de ahorro de trabajo y de recursos necesarios, y en definitiva de la reducción del "ruido" que las universidades, como complejas máquinas burocráticas, acaban generando entre sus usuarios. Amén de una potencial mayor transparencia de sus procesos y decisiones, dado el mayor caudal informativo que puede situarse al alcance de los usuarios o del público en general.

Aun cuando no existen estudios definitivos que orienten los parámetros específicos de la "calidad de una universidad virtualizada", en virtud que la "gestión general" está implícita en cada uno de los procesos que subyacen en las funciones sustantivas universitarias, se requiere que cualquier iniciativa o desarrollo de las TIC en el escenario universitario esté sustentada por una adecuada y bien concebida estrategia de administración y gestión no sólo de las tecnologías y procedimientos sino de la actuación de los actores y de la interacción de los mismos con las innovaciones que se generen y con los sistemas comunicacionales que se establezcan en lo interno y en la vinculación con el entorno.

Ello lleva a plantear la necesidad de ir más allá del aspecto técnico y pedagógico de la virtualización de la enseñanza, y señalar que este proceso es sobre todo un asunto cultural que implica una redimensión y desplazamiento en la manera de concebir el comportamiento y estructuras organizacionales que hasta ahora vienen siguiendo las universidades venezolanas, no sólo en sus relaciones internas, sino en su relación con la sociedad y los compromisos de eficiencia e inmediatez que acompañan los entornos caracterizados por la interactividad de "Ios servicios educativos virtualizados".

Supone entonces, por una lado la transformación en el interior del gobierno de cada institución, y por otro, la acción consensuada entre las distintas instituciones que conforman el sistema a fin de equilibrar los esfuerzos, recursos y logros hasta ahora alcanzados de manera individual, teniendo presentes las necesidades y demandas del conjunto para ofrecer resultados y respuestas adecuadas que permitan atender las demandas cada vez más complejas, de manera efectiva, $y$ de esta manera reforzar su liderazgo ante la sociedad.

De allí que en un escenario donde se espera que la universidad hará un gran esfuerzo por apoyar su gestión al máximo en el uso de las TIC en un enfoque virtual, Ruiz Bolívar (s/f), plantea que,

(...) es importante tener presente que, más allá del aspecto tecnológico, la fundamentación de la transformación universitaria, depende básicamente de: (a) la visión compartida que tengan los 
miembros de la comunidad académica acerca de un nuevo modelo universitario que responda a las expectativas de cambio a que aspira dicha comunidad, así como la sociedad global; (b) el compromiso para su instrumentación, asumido por los diferentes actores institucionales, orientados por genuinos valores académicos; y (c) las acciones estratégicas emprendidas por el liderazgo universitario, como responsable de guiar el proceso de transformación.

Es necesario entonces, que la virtualización de la universidad se entienda como la potenciación de al menos tres dimensiones: una nueva cultura de la educación, que reinventa la enseñanza y el aprendizaje; nuevas formas de conmensurabilidad, que consolida la interactividad, la conectividad y los colectivos inteligentes como estrategias para crear comunidades virtuales de aprendizaje; y finalmente de nuevas formas de organización institucional, que obligan a reformular las coordenadas espacio-temporales de esa "organización" Ilamada universidad.

Desde esta perspectiva, tal como refieren Carrillo, Piñero y García (2004) el gobierno electrónico no sólo se constituye en una herramienta que permitirá incorporar valor a las funciones universitarias hasta ahora desempeñadas, a través del uso intensivo de las TIC, sino que plantea la posibilidad de ser utilizada como una estrategia de gestión para alcanzar la modernización universitaria en relación con la necesidad de una mayor excelencia y transparencia en la gestión de sus funciones, así como la búsqueda de una mayor eficiencia y mejoramiento de la calidad, responsabilidad y democra- tización en la prestación de los servicios y su vinculación con la sociedad.

\section{El gobierno electrónico y la modernización de la gerencia pública}

En la década de los noventa, las actividades relacionadas con las TIC se han vinculado estrechamente con el crecimiento económico y el progreso social, de manera que es observable como los distintos niveles de gobierno han ido asimilando, a diferentes ritmos y grados en cada país, que la difusión de estas tecnologías constituye un elemento generador de externalidades positivas en el conjunto económico y social así como que ofrece nuevas oportunidades para la evolución de las estrategias de relación entre ciudadano y gobierno. Lo cual ha marcado claramente el desarrollo y la extensión de la utilización de las TIC en determinados segmentos del entramado económico y social.

Las esferas gubernamentales no podían quedar al margen de este proceso. Al respecto Jordana (2001) plantea como los gobiernos a nivel mundial han transitado de manera muy diferenciada, por distintas acciones e iniciativas que procuran incorporar en uso intensivo de las TIC en los distintos ámbitos de los gobiernos, y de esta manera atender las nuevas necesidades de democratización, rendición de cuentas, transparencia de las administraciones públicas.

Es así como surge el gobierno electrónico. A grandes rasgos puede decirse que el gobierno electrónico consiste en el uso de las nuevas tecnologías de información y comunicación con el fin de pro- 
mover un gobierno más eficaz y eficiente, haciendo los servicios gubernamentales más accesibles a la ciudadanía, permitiendo un mayor acceso a la información, todo esto con el propósito de transformar al gobierno en una institución competitiva y orientada al ciudadano.

Como concepto comenzó a ser utilizado desde la segunda mitad de los noventas para dar cuenta de las transformaciones que produce la incorporación de tecnologías en el quehacer de las instituciones públicas; específicamente en el año 1999 se inicia en el mundo el desarrollo del concepto de gobierno electrónico. Al respecto, el Gartner Group en 2000 (cit.por Gascó, 2003) especifica que el gobierno electrónico se refiere a "the continuos optimization of government service delivery, citizen participation and governance by transforming internal and external relationships through technology, the Internet and new media".

Según Dinsdale (2002) el gobierno electrónico es el estado en que los gobiernos son interactivos, interjurisdiccionales, totalmente conectados a los ciudadanos, trabajando conjuntamente en los temas y encontrando soluciones a políticas y programas de manera coherente y democrática. Por su parte, el Banco Mundial (2003) ha definido al gobierno electrónico (e-Gov) como "los sistemas de información y las tecnologías de información y de comunicaciones (TIC's) de los gobiernos, que transforman las relaciones con los ciudadanos, el sector privado y otras agencias estatales para promover el crecimiento del ciudadano, mejorar la entrega de los servicios, consolidar responsabilidades, aumentar la transparencia, o mejorar la eficacia del gobierno".
Otras definiciones apuntan hacia la utilización de distintas herramientas y aplicaciones de las nuevas tecnologías de información y comunicación, por la administración pública como vía para la interconexión con otras organizaciones y personas; así lo plantea la OCDE (1998; cit por Criado, Ramilo y Serna, 2002), cuando define al gobierno electrónico como la aplicación de tecnologías basadas en Internet para actividades comerciales y no comerciales en el seno de las administraciones públicas. Igualmente Holmes (2001) y la ALADI (2003) refieren que el gobierno electrónico, o e-gobierno, significa el uso estratégico e intensivo de las TIC en particular de Internet para ofrecer servicios públicos de una manera más adecuada, convenientemente, orientada a los consumidores, económica y en general más efectiva.

Por otra parte, según Gallegos (s.f.), el gobierno electrónico en Venezuela significa el aprovechamiento de las TIC para aumentar la inclusión de amplios sectores que han estado al margen de la acción social del Estado. Dichas Tecnologías no sólo pueden propiciar la transformación del Estado, maximizando la eficiencia de la administración pública, también son un mecanismo para aumentar la transparencia y garantizar la seguridad de la nación.

Por lo que, el concepto del Gobierno Electrónico en Venezuela está fundado en la estructura de Red en la cual la interdependencia, las relaciones no lineales y los procesos cíclicos fundamenten la acción de los funcionarios públicos y de los organismos públicos, para lo cual las TIC representan un excelente dispositivo dada su estructura lógica, sus patrones 
de funcionamiento y las características de sus elementos constituyentes. De allí, que está orientado a desarrollar reformas en áreas prioritarias como la gerencia interna, la administración pública, los servicios públicos y la información pública a través de la suplantación, el apoyo y la innovación sobre los procesos encargados a la administración pública.

De hecho, una estrategia de esta naturaleza se enmarca en los lineamientos legales y de política del Estado Venezolano, a partir de la publicación el 10 de Mayo del año 2000 del Decreto 825, que establece el uso y promoción de la Internet por parte de los entes y órganos de la Administración Pública. el cual amplía y detalla el marco regulatorio sobre las Tecnologías de Información y Comunicación, que se establece en el artículo 110 de la Constitución de la República Bolivariana de Venezuela, la Ley Orgánica de Ciencia, Tecnología e Innovación, la Ley Orgánica de Telecomunicaciones, El Artículo 12 de la Ley Orgánica de la Administración Pública, la Ley de Delitos Informáticos, el Decreto con Fuerza de Ley de Simplificación de Trámites Administrativos y el Decreto con Fuerza de Ley sobre Mensajes de Datos y Firmas Electrónicas.

En resumen, el gobierno electrónico es el uso que hacen, los órganos de la administración pública, de las modernas tecnologías de la información y comunicaciones, en particular Internet, para mejorar los servicios e información ofrecidos a los ciudadanos, incrementar la eficiencia y la eficacia de la gestión pública, proveer a las acciones del gobierno un marco de transparencia y crear mecanismos para facilitar la participación ciudadana en la toma de decisiones de la gestión pública. Su funcionalidad es derivar por medio de las TIC servicios a una comunidad determinada, actividades y servicios que de hecho ya están incorporadas a las competencias de un gobierno, ya fuere nacional, regional o local.

Podemos decir entonces, que el gobierno electrónico no es la tecnificación de la burocracia existente, sino que tiene que ver con la inserción de la creatividad y la innovación en la Administración Pública, para crear procedimientos asociados a viejos y nuevos servicios, que resuelvan y simplifiquen la vida del ciudadano (Berrizbeitia, s.f.).

Ello significa que el gobierno electrónico está orientado a un proceso de mejora y modernización de la gestión y gobernabilidad de las instituciones públicas por cuanto está implícita la transformación profunda y compleja de los procesos y estructuras creadas para la oferta electrónica de los servicios gubernamentales. Tal como lo plantean Alcántara y Cendrós (2005) depende funcionalmente de muchas aristas o ramificaciones como el compromiso de los gerentes públicos, la dinámica política, los servicios civiles y las funciones parlamentarias y judiciales; se requiere que el gobierno tenga apertura a un modelo de prestación de servicios bastante complejo, en el que interactúan tanto los niveles como las aristas del sistema de gobierno.

El gobierno electrónico está todavía en desarrollo, sin embargo, parece cada vez más evidente la existencia de una tendencia internacional hacia su implementación, incluso en aquellas administraciones públicas en las que existe un largo camino por recorrer y las barreras 
son más intensas (Criado, Ramilo y Serna, 2002). De hecho, son varias las instituciones públicas nacionales, regionales y locales en Venezuela que han iniciado un proceso de implantación de gobierno electrónico, tales como el SENIAT, el CNE, el Poder Judicial, y algunos municipios, los cuales pueden ser visitados en la página http://www.gobiernoenlinea.gob.ve. De las varias instituciones que aparecen en el portal, solo se señala a la Universidad Nacional Abierta como única referencia de educación superior.

Por otra parte, en un estudio realizado por Gómez (2007) sobre "El desarrollo del gobierno electrónico en el municipio venezolano", hace referencia que en términos generales el gobierno electrónico municipal en Venezuela se encuentra entre las etapas de interacción y transacción, con algunos casos que incursionan en atributos de democracia electrónica, destacando las alcaldías de Valencia, Chacao, Los Salias, Baruta, AIcaldía Mayor, San Cristóbal, Pedro María Freites, Naguanagua y Maturín (pág. 78).

A estas iniciativas, se suma el borrador del proyecto de Ley de Infogobierno del Ministerio de Ciencia y Tecnología (2006), la cual tiene como objetivo el establecimiento de las normas, principios y lineamientos aplicables a las tecnologías de información que generen y utilicen los sujetos a que se refiere el artículo 4 de esta Ley, con el fin de mejorar la gestión pública, hacerla transparente, facilitar el acceso de las personas a los servicios y a la información pública en su rol de protagonista y contralor, utilizar dichas tecnologías como herramienta de apoyo para la lucha contra la burocracia y la corrupción además de promover el desarro- llo endógeno y garantizar la soberanía tecnológica, el progreso y bienestar de la nación. En este primer borrador llama la atención que en el art. 4, numeral 8 , se reconoce a las universidades públicas como sujetos aplicables de la ley.

\section{La gerencia de la virtualización universitaria: premisas desde una estrategia de gobierno electrónico}

La universidad es un espacio, en el cual se desarrollan políticas y estrategias que conllevan a alcanzar una mejor educación a los ciudadanos, con el propósito de incrementar el desarrollo del país, resulta impostergable promover una mayor eficiencia en estas instituciones. Por esto, se requiere entre otros elementos, el incremento de la pertinencia social de las instituciones universitarias, el mejoramiento continuo de las funciones de docencia, investigación, extensión y producción dentro de una gestión dinámica y participativa que permita elevar la excelencia académica.

La universidades al igual que muchas instituciones públicas y privadas prestadoras de servicios, están siendo presionadas por una ciudadanía que ha comenzado a cuestionar la forma tradicional de hacer las cosas, por lo que no deja de ser significativa la consideración de las TIC como herramienta con enorme potencial para configurar estructuras organizativas y modelos de gestión que permitan ofrecer una respuesta única, ágil, eficiente, de calidad y transparente para todos los miembros de la comunidad académica y de los ciudadanos en general. De esta manera, tal como refieren 
Criado, Ramilo y Serna (ob. cit) el uso de las TIC puede constituirse en un motor de cambio, que vaya más allá de la modificación de los elementos técnicos (tecnología, estructuras, procesos, entre otros) sino también en lo que es más importante: el cambio en los valores y comportamientos que configuran la cultura organizativa existente.

El concepto de "universidad virtual" cada día gana más cuerpo, y hoy por hoy representa una de las tendencias modernizadoras de mayor impacto en las Instituciones de Educación Superior. De hecho la complejización y ampliación de los procesos y servicios educacionales ofrecidos en un campus virtual ha supuesto una verdadera reconfiguración y reingeniería organizacional e institucional en el seno de las universidades que desarroIlan el aprendizaje en línea.

Por campus virtual se entiende una metáfora del entorno donde se desarrolla el proceso educativo gracias a la utilización de las nuevas tecnologías y consiste en un programa informático lógico que en forma integral es utilizado como medio y ambiente en el cual se ofrecen las carreras y cursos universitarios. Desarrollar y ofrecer tal proceso implica a la par una visión de la gestión académica diferente a la tradicional.

Es por ello, que la evolución y el mejoramiento de los mismos fue haciendo que las universidades "virtualizaran" otros procesos paralelos de apoyo a la gestión académica, como es la gestión administrativa. Asimismo, el carácter cada vez más especializado de los usuarios (actores académicos y ciudadanía en general) ha ido exigiendo también no sólo mejorar la calidad de la información aca- démico-administrativa, sino una mayor inmediatez, sencillez y transparencia en la prestación y tramitación de las mismas.

Siendo que el gobierno electrónico, según el Programa de Naciones Unidas para el Desarrollo (PNUD) no es más que "aquellas actividades basadas en las TIC y en particular Internet, que el Gobierno desarrolla, para aumentar la eficiencia de la gestión pública, mejorar los servicios ofrecidos a los ciudadanos y proveer a las acciones de Gobierno de un marco más transparente". Se considera, que el mismo puede ser implementado como estrategia de innovación, modernización y transformación de la gestión universitaria, en virtud de las dimensiones de cambio, excelencia y mejoramiento no sólo de los productos y procesos académico-administrativos que se dan en los entornos educativos virtuales, sino también de la participación e integración de los actores y entre los actores que establecen vínculos en tales espacios.

Según Silvio (2000) hay variaciones importantes entre distintos tipos de universidad en relación con la disponibilidad, el acceso y el uso de tecnologías asociadas a la virtualización, de esta manera, se cuenta con universidades presenciales, universidades a distancia tradicionales y universidades totalmente virtuales. En el caso del sistema de educación superior venezolano nos encontrarnos con instituciones ubicadas en el primer y segundo caso, las cuales poseen en su mayoría portales en la web, con diferentes grados de interactividad y uso de las TIC como parte de sus procesos de gestión académica administrativa.

Ello lleva a la siguiente pregunta: en el entorno de virtualidad de la educa- 
ción superior ¿es posible lograr la innovación y modernización de la gestión universitaria a través del gobierno electrónico? La respuesta es que si lo es, atendiendo a las siguientes premisas estratégicas:

- Liderazgo: Debe existir un liderazgo político fuerte y situado al más alto nivel, para conseguir superar las resistencias al cambio, mover las voluntades de todos los actores de la comunidad universitaria de manera consensuada y garantizar la implantación horizontal y transversal del proyecto de cambio.

- Visión y compromiso compartido para el cambio, asumido por los diferentes actores institucionales y orientados por los genuinos principios y valores académicos.

- Discusión, desarrollo, elaboración e implantación de un proyecto institucional estratégico de innovación tecnológica para el gobierno electrónico que implicaría:

- Conocimiento de los requerimientos, necesidades y demandas de los usuarios internos y externos a la institución.

- Análisis y definición de la cadena de valor institucional, es decir de los productos, procesos, servicios, in- formación y otras relaciones comunicacionales de tipo interactivo que la institución desee ofrecer a corto, mediano y largo plazo (qué es lo que crea valor a lo que hace la institución, como se puede mejorar y cómo se relaciona con otros factores creadores de valor).Asimismo, se requiere identificar la cadena de valor según el estadio de gobierno electrónico ${ }^{2}$ en el que se encuentre la institución universitaria y la cadena de valor deseable al máximo estadio.

-Desarrollo o adquisición (según sea el caso), transferencia y evaluación tecnológica de una plataforma tecnológica con las capacidades necesarias para sostener la red de interactividad deseada. $Y$ paralelamente, una infraestructura física y lógica que permita el funcionamiento de los servicios telemáticos y los servicios de información y comunicación, mediante el diseño de una arquitectura lo más amigable posible.

- Cualquiera sea la tecnología para la arquitectura del gobierno electrónico tanto en plataforma como de software, es necesario que los componentes y aplicaciones sean reutilizables, flexibles, abiertas (integra-

2 Al respecto, la Red de Administración Pública de las Naciones Unidas (UNPAN, cit.por Pocoví y Farovollini,2002), propone cinco categorías que identifican el nivel en que se encuentra el Gobierno Electrónico en cada país o región:

1. Presencia emergente en línea (información estática)

2. Presencia mejorada en línea (información más dinámica y con opciones)

3. Presencia interactiva en línea (intercambio de información formal entre el usuario y el Gobierno)

4. Presencia transaccional en línea (acceso fácil a los servicios, priorizados por el usuario)

5. Presencia totalmente integrada en línea (portal único que incluye el total de los servicios). 
bles), escalables, performantes (tiempo de respuesta adecuado), disponibles (en forma permanente), con tolerancia al error (éstos no deben general el colapso del sistema en su conjunto) y actualizables (con facilidades para ello).

-Análisis, planificación, organización, dirección, implementación y evaluación compartida (horizontal y vertical) de un programa de reingeniería de todos procesos y procedimientos vinculados con las funciones universitarias y actividades de gestión académico-administrativas que pasarán a la red de interactividad electrónica. Ello permitiría la configuración de un programa de soluciones tecnológicas a cada uno de los procesos y macroprocesos y su respectiva transferencia horizontal.

-Desarrollo de procesos de consulta, asimilación, capacitación permanente, información, validación y desarrollo de competencias en cada uno de los miembros de la institución y a todos los niveles en relación del impacto de la tecnología incorporada no sólo a nivel técnico, sino en la "nueva manera de hacer las cosas", esto es en cuanto a procesos, procedimientos y toma de decisiones de tipo académico-administrativo.

- Implicación permanente y continua en el proyecto de todos los actores y miembros internos de la institución universitaria (autoridades, docentes, estudiantes, empleados, obreros, gremios, asociaciones estudiantiles, docentes, entre otras) así como de otros actores vinculados, tales como otras organismos del Estado (Ministerio de Educación Superior, OPSU, Ministerio de Finanzas, entre otros), instituciones de la banca privada y pública, empresas proveedoras de servicios, otras instituciones de educación superior, instituciones de educación media y diversificada, entre otras.

- Asignación y disponibilidad de los recursos económicos, pero también recursos en términos de conocimiento, formación y capacitación técnica de los miembros de la comunidad universitaria y autoridades medias y altas, así como recursos éticos fomentando una cultura de servicio público.

- Conformación de un equipo(s) de trabajo multidisciplinario, dándole jerarquía organizativa para la planificación, ejecución y evaluación permanente del proyecto institucional estratégico de innovación tecnológica para el gobiemo electrónico. Este equipo debe mantener una comunicación abierta y fluida con todos los principales actores de los procesos involucrados.

- Una vez lograda la implantación del proyecto es necesario configurar una unidad organizativa ubicada al más alto nivel que permita el continuo seguimiento y mejora del mismo. Esta unidad estará conformada por personal multidisciplinario especializado relacionado con cada uno de los procesos y productos que se desarrollen virtualmente. Sin embargo, es posible que la conformación organizativa de 
este equipo de personas se ajuste a las necesidades propias de cada universidad. Igualmente, debe mantener canales abiertos y fluidos de comunicación.

- Diseño, implementación y actualización de estándares de calidad del servicio prestado, lo cual debe representar un esfuerzo de capacitación e intercambio de información entre los miembros de la unidad y equipo de personas directamente vinculadas al funcionamiento del gobierno electrónico.

- Seguimiento permanente de las expectativas de los usuarios tanto para orientar la agenda de los servicios como para desarrollarlos.

- Es necesario paralelamente realizar un proceso de mejora de los marcos regulatorios y normativos que sustentan el funcionamiento académico-administrativo de la institución universitaria, ya que es imposible implantar nuevos mecanismos exitosos de Gobierno electrónico si se sigue trabajando con los procesos y procedimientos complejos, excluyentes, obsoletos e ineficientemente burocráticos que han caracterizado a las universidades públicas especialmente. Ciertamente, tal como lo afirma Berrizbeitia (ob. cit.), el Gobierno Electrónico no es la tecnificación de la burocracia existente, tiene que ver más bien con la inserción de la creatividad y la innovación en la administración pública para crear procedimientos asociados a viejos y nuevos servicios, que resuelvan y simplifiquen la vida del ciudadano, y en este caso de cualquiera de los miembros de la comunidad universitaria, haciéndola a su vez más eficiente y con mayor calidad.

- Incorporación paulatina de los estudiantes a la era de la telemática, mediante la utilización de la web como instrumento de comunicación y de aprendizaje.

Son incontables las posibilidades y el abanico de oportunidades que el gobierno electrónico traería a la modernización de la gestión universitaria. En este sentido, tanto los requerimientos como los factores deben ser integrados al proceso de una cultura tecnológica, adecuada al entorno en la cual la tecnología debe formar parte integral del pensamiento y acción del potencial humano en la búsqueda de una universidad más cercana, más productiva y competitiva. Una universidad de servicio, que facilite los procesos de intercambio e interacción entre todos los actores que de una u otra forma tienen que ver con ella.

En el Cuadro 1, se ofrecen algunos de los servicios que pueden ser asumidos en una estrategia de gobierno electrónico a nivel de las instituciones de Educación Superior y su relación con el entorno.

Asimismo, entre las informaciones y las herramientas que pueden disponerse están (Carrillo, Piñero y García, 2004):

- Información: La Universidad: Misión, Visión, Historia, Organigramas, Decanatos, Oportunidades de estudio, Admisión, Proceso de inscripción, Calendario académico, Registro de usuarios, Asignación de login y password, Reglamentos, Gacetas Universitarias, Aranceles, Programas 


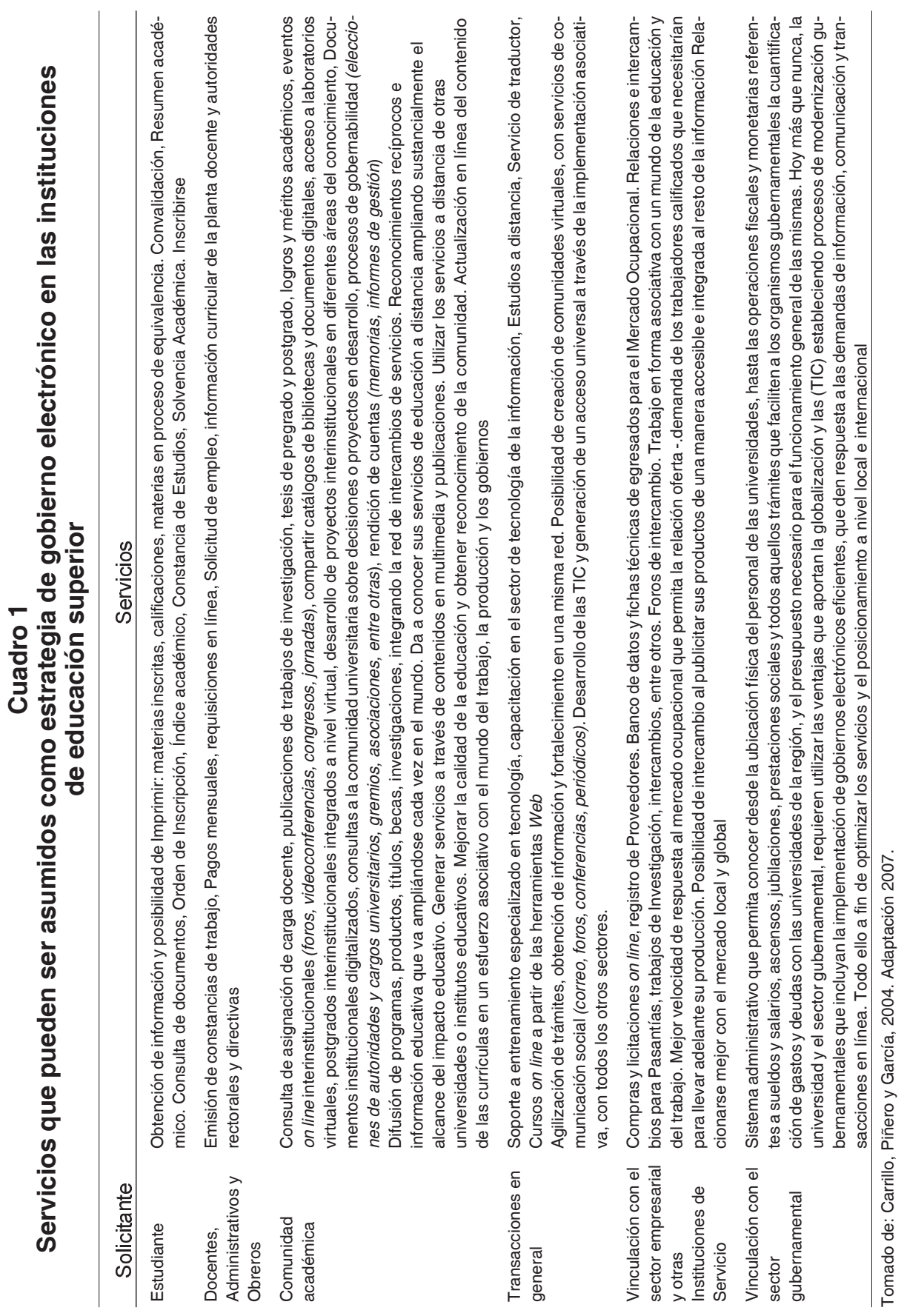


de Bienestar social, entre los que destacan Becas, Asociaciones estudiantiles; Catálogos automatizados, Bases de datos bibliográficas y de Revistas. Cabe destacar que estos aspectos conforman por lo general la Fase de Presencia en un proyecto de gobierno electrónico, razón por la cual todas la universidades que tienen sitios y portales en la Web ya presentan esta información, así como algunas herramientas y servicios posteriormente señalados, vinculados con inscripciones y estudios virtuales De hecho, a través de la red UNIVERSIA es posible visualizarla de manera conjunta e interinstitucional.

- Herramientas interactivas: Para lograr la interactividad con los diferentes usuarios se desarrollaran herramientas web, de acuerdo con los diversos servicios que ofrecerá la institución universitaria. Destacan los siguientes: documentos electrónicos o digitalizados, firmas digitalizadas, foros, chats, direcciones de contacto a través de correo electrónico, lista de correo, grupos de trabajo, planillas y pagos on line, eventos on line, entre otros.

\section{A manera de cierre}

El mundo actual enfrenta inevitablemente un impetuoso proceso de cambio que incide en casi todas las áreas y estructuras de cada sociedad, sin importar cuál sea su nivel de desarrollo o de subdesarrollo. La experiencia de las organizaciones y las naciones contemporáneas que han tenido éxito en alcanzar sus misiones fundamentales, indica que en cada caso el uso apropiado de las TIC ha sido un factor determinante en el fortalecimiento de sus capacidades para enfrentar los problemas que surgen de la complejidad del mundo actual. El resultado de estos procesos ha convertido a las TIC en instrumentos transversales a la sociedad, es decir, penetran y se integran prácticamente en todas las actividades y sectores de la misma, y hoy no es posible prescindir de ellas, puesto que pautan el tiempo, la manera de trabajar, aprender, comunicarse y en consecuencia la manera de gerenciar.

Ante este escenario en constante cambio, la "Sociedad de la Información", como la llaman muchos, ha debido estructurar cambios en todos sus niveles, entre los que destaca por supuesto la Educación Superior. Como institución social constructora de saberes y formadora de ciudadanos, ésta no debe ni puede escapar a las nuevas tecnologías y formas de comunicación, negando su inevitable integración que ya penetrado hasta los más impensables resquicios en la generación y divulgación de conocimientos.

El cambio debe ser entonces una necesidad para las Instituciones de Educación Superior ya que están actualmente, más que nunca, expuestas al cambio y a la incertidumbre. De hecho, a las dificultades por conocer el objeto de la intervención (¿qué valores transmitir?, ¿para qué sociedad preparamos a los estudiantes?, entre otros.), se añaden los problemas que afectan a su propia existencia (aumento de la matrícula estudiantil, deterioro y insuficiencia de la planta física, demandas de nuevas políticas gubernamentales, limitaciones presupuestarias, 
entre otras.). Si es así, tiene sentido pensar que en este escenario sólo las instituciones educativas capaces de renovarse $y$ reinventarse de forma continua, que tienen unas estrategia ante el permanente cambio del entorno, lograrán los niveles de calidad, pertinencia y excelencia que cada día exige la sociedad.

Las TIC se constituyen en un aporte constructivo a los nuevos requerimientos de la educación superior basada en la calidad, donde la gestión pedagógica y la gestión del conocimiento deben ser transversales a todas las funciones máximas y de razón de ser universitaria. Es por ello que, la virtualidad le ofrece a las instituciones de educación superior la posibilidad de crear entornos nuevos de relación en el desarrollo de los procesos de docencia, investigación, extensión y producción, y como tales, deben de ser tratados de forma distinta para extraer de ellos el máximo de su potencial. La riqueza de estos nuevos entornos, todavía en fase de exploración, es enorme y su poder reside en la capacidad de saber usarlos al máximo de sus posibilidades, pero para ello es necesario cambiar de hábitos, ser creativos, para rendir en este nuevo medio mientras se pueda hacer.

No obstante, el desarrollo tecnológico y la compleja dinámica del mundo actual, pudiesen permitir representar el uso de las TIC, de manera extraordinaria y perfecta, pero se puede correr el riego de que se vuelva incontrolable. En este sentido la incorporación de las TIC y de forma especial el gobierno electrónico en las universidades debe ser el resultado de una formación adecuada de todos sus miembros y de una planificación y gerencia en consonancia con la misión, visión y valores de la universidad y de la proyección que la misma desee abarcar en respuesta a la sociedad donde se encuentre anclada.

Efectivamente, Silvio (1999) señala que las universidades de los países en desarrollo enfrentan el desafío de servir a una población cada vez mayor de estudiantes, más diversificada social y culturalmente, en un nuevo ambiente social, más dinámico y turbulento. La virtualización (parcial o total) de estas organizaciones puede ser un factor transformador de sus estructuras y funciones, un instrumento para mejorar su cobertura, calidad, pertinencia y equidad de acceso y una manera de construir una nueva identidad en la sociedad del conocimiento.

No se trata de que las universidades presenciales dejen de existir o cambien radicalmente sus modalidades de enseñar 0 aprender, sino de ampliar las posibilidades de acceso al conocimiento científico y a la cultura universitaria a lugares geográficos que de la manera tradicional, sería casi imposible o demasiado costoso.

Sin embargo, más allá de la virtualización de la enseñanza universitaria, organizar la educación en la virtualidad requiere de una estructura particular. Al igual que cualquier otra organización educativa, las instituciones educativas virtuales deben gestionar tanto los procesos que afectan a los estudiantes (organización académica) como los que afectan a los docentes (administración de los profesores-facilitadores) y a los servicios académico-administrativos (administraciones de los entornos virtuales de aprendizaje), previendo siempre que el tipo de gestión no sea más de lo mismo de los sistemas presenciales. 
Es por ello, que en el marco de las nuevas políticas del estado venezolano de modernización de las instituciones públicas mediante el uso de las TIC, el gobierno electrónico se presenta como una herramienta de oportunidad para la innovación y transformación de las universidades, ya que ofrece vías para la introducción de mejoras en la acción de su gestión académico-administrativa. Mejoras que, aunque limitadas, es bueno no prescindir de ellas si se quiere avanzar hacia la modernización; pero como ya se ha comentado en puntos anteriores, no pueden sustituir la acción y el compromiso político de cara a la consolidación institucional de la calidad, pertinencia y excelencia en el sistema de educación superior.

Para ello se plantea, que una estrategia institucional universitaria de gobierno electrónico debe partir de algunas premisas que se orientan fundamentalmente hacia la visión estratégica, la infraestructura tecnológica, automatización de procesos, uso de redes y de Internet, capacitación permanente e inteligencia para la gestión. Por lo que es necesario tener en cuenta que los obstáculos aparecen cuando está presente el mito que la modernización se alcanza sólo por la vía la informatización; cuando falta liderazgo de la autoridad superior; cuando el foco es principalmente tecnológico ignorando la importancia de las personas y de los procesos; cuando existen debilidades en la gestión de los proyectos y desconocimiento del cambio organizacional que involucran; cuando se hace omisión voluntaria de los valores éticos y morales que deben ser una constante, cuando se carece de enfoque hacia el usuario, en este caso de la comunidad universitaria y falta la presencia de la sociedad en general.

Es por ello, que a juicio de Tesoro (2007),

Las experiencias de gobierno electrónico en la mayor parte de los países de América Latina y del Caribe exhiben notorias limitaciones para lograr los beneficios, dado que su concreción requiere -más que incorporar tecnologías de información y comunicación (TIC)- plasmar profundos cambios institucionales y culturales que requieren nuevos valores, actitudes, conocimientos, capacidades y habilidades, trayendo consigo exigentes requerimientos de formación y capacitación de los agentes del Estado, así como una activa difusión y orientación entre los diversos actores sociales (pag 44).

Ello significa, que es necesario y prioritario contar con una planificación coordinada que afecte a todos los niveles de la institución universitaria y que vaya más allá del simple proceso de informatización de un expediente académico-administrativo. Para el aumento de la eficacia y la eficiencia de la gestión universitaria a través de las TIC hace falta reconsiderar cualitativamente las formas de organización del trabajo, las relaciones humanas en su seno y, consiguientemente, las estructuras de poder dentro de la propia institución.

De ahí la importancia de insertar las estrategias de introducción de iniciativas de gobierno electrónico en los planes estratégicos de modernización y transformación institucional. El rediseño de procesos, la definición de puestos de trabajo, los sistemas de formación, las estructuras de incentivos que se introducen a tra- 
vés de las iniciativas de orden académico-administrativo-electrónica, deben ser planificados de forma global, en función de cada cultura organizativa y atendiendo tanto a las demandas externas como a las necesidades que se plantean dentro de la propia estructura académico-administrativa. Sólo así el desarrollo de iniciativas de gobierno electrónico puede constituirse como una oportunidad para la introducción del cambio e innovación organizativa.

Es así como lo plantean Pocoví y Farovollini (2002), al referir que todo proceso de transformación exitoso de gobierno electrónico "pasa por reconocer la importancia y trabajar sobre los cuatros elementos que constituyen la base de dicho proceso: La gente, los procesos, el marco regulatorio y la plataforma tecnológica"... por ello, "Si una organización va a inclinarse hacia este nuevo paradigma, debe tener en cuenta que la gestión social es tanto o más importante que la gestión técnica. Así, se podrá generar un cambio genuino en esa dirección (...)".

También resulta indispensable tomar en cuenta, la acogida que las TIC han tenido en el mundo empresarial y en la sociedad en general, lo cual debe tomarse como punto de reflexión y consideración por parte de la universidad, para no seguir llegando tarde al encuentro de oportunidades. En este sentido, la flexibilidad, la información, la comunicación, el rol del docente, el papel del alumno, la gerencia, los valores y la apertura al cambio son sólo algunos de los elementos que podrian servir de referencia para considerar las premisas presentadas como estrategia de gobierno electrónico.
Esto sin perder de vista que la educación, en este escenario globalizado, globalizante y postmoderno se ve cada vez más comprometida y requiere que se tome conciencia de la imperiosa necesidad de emprender procesos educativos basados en las TIC encaminados a fortalecer el tejido social, con el consecuente incremento del grado de cohesión entre las poblaciones. Desde esta óptica, parece más necesario que nunca, el gobierno electrónico, como plataforma para EDUCAR a una ciudadanía apoyada en valores democráticos y en un sentimiento de unidad y solidaridad.

Todo ello conduce a finalizar afirmando que los retos que debe enfrentar la institución universitaria ante la implantación de proyectos de gobierno electrónico van más allá de facilitar el cambio tecnológico. La cultura de la calidad, eficacia y eficiencia, de orientación al usuario o comunidad universitaria, de transparencia y responsabilidad sólo será posible si es asumida como tal por todos y cada uno de los miembros de dicha comunidad, si se consigue, en suma, el cambio y la innovación institucional universitaria estará tal vez más cerca de ser alcanzada.

\section{Referencias Bibliográficas}

ALADI (2003). Estudio sobre Gobierno Digital. Secretaria General. (s.m.r.). Disponible al lector interesado.

Alcántara, Albino y Cendrós, Jesús (2005). Modelo de gobierno electrónico para la alcaldía rural del Municipio Autónomo Colón. Revista Electrónica de Estudios Telemáticos. Volumen 4 Edición No. 2. Documento en línea Disponible en: http://www.urbe.edu/ 
telematica/articulos.html Consulta: 2006, Julio 22.

Angulo, Martín Pastor (2000). Perspectivas de las nuevas tecnologías en educación a distancia para el siglo XXI: interactividad y virtualización. La Revista del Doctorado, Año III, No. 7, Abril.Mexico. Doctorado en Ciencias Sociales UAS-UNISON-UABC-CIAD. Documento en línea Disponible en: http:// www.uasnet.mx/dcs/revista.html Consulta: 2006, Julio 22.

Backus, Michael (2001). E-governance in Developing Countries del Instituto Internacional para la. Comunicación y el Desarrollo de Holanda, IICD, Research Report N. 3, April 2001. Documento en línea. Disponible en http: www.ftpiicd.org/files/research/reports /report3.pdf Consulta: 2006, Octubre 25.

Banco Mundial (2003). Gobierno de la Provincia de Misiones. (e-gov). Documento en línea Disponible: http://www. misione.gov.ar/egov. Consulta: 2004, Julio 18.

Berizibetia, Jorge (s.f.) Implementando el Gobierno Electrónico Documento en línea Disponible en: http://www.gobiernoenlinea.gob.ve/directorioestado/gob _electronico_02.html. Consulta: 2006, Agosto 31.

Carrillo, Alfonso; Piñero, María Lourdes y García G, Blanquita C. (2004). Red virtual universitaria de la región Centro-Occidental: Una estrategia integrada de gobierno electrónico para la Educación Superior. Revista Ciencias de Gobierno Julio-Diciembre. Año 12, No.16.Maracaibo. IZEPES. Gobernación del Edo. Zulia.

Castells, Manuel (1997). La Sociedad Red: La Era de la Información Vol. 1. España: Alianza Editorial

Criado G., J. Ignacio, Ramilo A., María Carmen y Serna, Miguel Salvador (2002). La necesidad de teoría (s) sobre gobierno electrónico. Una propuesta Integradora. XVI Concurso de Ensayos y Monografías del CLAD sobre Reforma del Estado y Modernización de la Administración Pública. "Gobierno Electrónico". Mención Honorífica. Caracas.

Curci La Rocca, Renata (2003). Diagnóstico de la educación superior virtual en Venezuela. Universidad Metropolitana/IESALC-UNESCO. Caracas. Documento en línea Disponible en: http://www.iesalc.unesco.org.ve/programas/internac/univ_virtuales/venezuela/vir_ve.pdf\#search=\%22educacion\%20virtual\%20en\%20venezuela\%22. Consulta: 2006, Agosto 30.

Dinsdale,Geoff.; et al ( 2002). Guía práctica para el Gobierno Electrónico : cuestiones, impactos y percepciones. BID. Centro Canadiense de Gestión. Documento en línea Disponible en: http://www.iberomunicipios.org /docs/guíagobelect.pdf. Consulta: 2004, Agosto, 14.

Gallegos, Arturo (s.f.) ¿Qué es gobierno electrónico? Documento en línea Disponible en: http://www.gobiernoenlinea. gob.ve/directorioestado/gob_electronico.html. Consulta: 2006, Agosto 31.

García, Blanquita C. y Pinto, Teodoro (2005). Las nuevas tecnologías de la información y comunicación y las funciones del profesor universitario. Revista Encuentro Educacional. Vol 12, № 3 Sep-Dic. 2005. pág. 415-441.

Gascó, Mila (2003). Impacto organizacional e institucional de proyectos de gobierno electrónico. Documento en línea Disponible en: http://www.congres.lluert.net/comunicacions/grup3/ Gasco.pdf. Consulta:2006, Agosto 15.

Gómez, Pavel (2007) El desarrollo del gobierno electrónico en el municipio venezolano. EnI@ce: Revista Venezolana 
de Información, Tecnología y Conocimiento. Año 4: No. 2, Mayo-Agosto 2007, pp. 67-80. Disponible en: http://dialnet.unirioja.es/servlet/revista?tipo_busqueda=CODIGO\&clave_ revista=7671 Consulta: 2007, Agosto 20.

Holmes, Douglas (2001). E-Gob. Estrategias para el uso eficiente del Internet en el gobierno. Mexico. McGraw Hill.

Jornada, Jacint (2001). Las administraciones públicas y la promoción de la sociedad de la información: opciones estratégicas y modalidades de intervención. Revista Gestión y Análisis de Políticas Públicas, CLAD. № 16.

Ministerio de Ciencias y Tecnología (2006). Ley de Gobierno electrónico. Borrador. Disponible en: http://tyto.ciens.ucv. ve/\%7Elbracci/leyti/ley-infogobiernomct-12-6-06.html Consulta: 2006, Agosto 12.

Pocoví,Gertrudis María Estela y Farovollini,Gustavo Ricardo (2002). Gobierno electrónico: un cambio estructural .La integración de la información como requisito. XVI Concurso de Ensayos y Monografías del CLAD sobre Reforma del Estado y Modernización de la Administración Pública "Gobierno Electrónico". Caracas. Documento en línea Disponible en: http:// www.cnti.gob.ve/cnti_docmgr/shared files/gobiernoelectronico5.pdf\#search $=\% 22$ plan $\% 20$ de $\% 20$ gobierno $\% 20$ electr\%C3\%B3nico\%22 Consulta: 2006, Agosto 15.

Rama, Claudio (2005). La Tercera Reforma de la Educación Superior en América Latina. Caracas. Convenio IESALC-UNESCO/IPAME.

Ruiz Bolívar, Carlos (s.f.). La Universidad venezolana en una época de transición. Documento en línea Disponible en: www.ucla.edu.ve/dac/investigaci \%F3n/compendium7/Epoca\%20de\%
20Transicion.htm. Consulta: 2006, Junio 17.

Silvio, José (1998). La virtualización de la educación superior: alcances, posibilidades y limitaciones. Revista Educación Superior y Sociedad. Vol 2. $\mathrm{N}^{\circ}$ 1. Caracas. Publicaciones IESALC/ UNESCO.

Silvio, José (2000). La virtualización de la universidad. ¿cómo transformar la educación superior con tecnología? Caracas. Ediciones IESALC/UNESCO.

Tesoro, José Luis (2007). La Red RIF-GE: articulación de conocimiento para el gobierno electrónico en las América. Enl@ce: Revista Venezolana de Información,Tecnología y Conocimiento. Año 4: No. 2, Mayo-Agosto 2007, pp. 43-66. Disponible en: http:// dialnet.unirioja.es/servlet/revista?tipo _busqueda=CODIGO\&clave_revista =7671 Consulta: 2007, Agosto 20.

Torres Albero, Cristóbal (s.f.) El impacto de las nuevas tecnologías en la Educación Superior. Un enfoque sociológico. Boletín de la Red Estatal de Docencia Universitaria. Vol 2. № 31. Documento en línea Disponible en: http:// www.uc3m.es/uc3m/revista/vol2num 3/Activos/pdfs/Torres.pdf\#search= $\% 22$ educaci\%C3\%B3n\%20superior\% 20y\%20las\%20TIC\%22 Consulta: 2006, Julio 14.

UNESCO (1997). Hacia una nueva educación superior. Actas de la Conferencia Regional Políticas y estrategias para la transformación de la educación superior en América Latina y el Caribe, realizada en Cuba del 18 al 22/11/1996. Caracas. Colección Respuestas. Ediciones CRESALC/ UNESCO.

UNESCO (1998). La educación superior en el siglo XXI: Visión y acción. Conferencia Mundial Sobre La Educación Superior. Documento en línea Dispo- 
Estrategia de gobierno electrónico en la gerencia de la virtualización universitaria

Piñero, Ma. Lourdes; Carrillo, Alfonso y García G., Blanquita C.

nible en: http://www.unesco.org/education/educprog/wche/declaration spa.htm Consulta: 2006, enero 30.

UNESCO (2001). XIVI Conferencia internacional de Educación. Ginebra 5 al 12 de septiembre de 2001. http://www.
ibe.unesco.org/internacional/IBEDDirector /dinotas.htm. [Consulta: 2007, enero 18]

UPEL (2003). Manual de Trabajos de Grado de Especialización y Maestría y Tesis Doctorales. Caracas: FEDEUPEL. 